\title{
Variability of the Ki-67 proliferation index in gastroenteropancreatic neuroendocrine neoplasms - a single-center retrospective study
}

\author{
Huiying Shi ${ }^{1}$, Qin Zhang ${ }^{2}$, Chaoqun Han $^{1}$, Ding Zhen ${ }^{1}$ and Rong Lin ${ }^{1 *}$
}

\begin{abstract}
Background: The Ki-67 index in gastroenteropancreatic neuroendocrine neoplasms (GEP-NENs) may change throughout the disease course. However, the definitive effect of Ki-67 variability on GEP-NENs remains unknown. The aims of this study were to evaluate changes in Ki-67 levels throughout the disease course and investigate the role of Ki-67 index variability in GEP-NENs.

Methods: Specimens with multiple pathologies were evaluated from 30 patients who were selected from 514 patients with GEP-NENs, being treated at Wuhan Union Hospital from July 2009 to February 2018. The Ki-67 index was evaluated among multiple specimens over the disease course. Univariable and multivariable Cox proportional hazards regression analyses were performed to assess the prognostic significance of various clinical and histopathologic features.

Results: Among the 514 patients with GEP-NENs, metastases were seen in 182 (35.41\%). Among the 30 patients from whom specimens with multiple pathologies were obtained, 24 were both primary and metastatic specimens and six were specimens collected over the course of the disease. Changes in Ki-67 levels were detected in $53.3 \%$ of the patients, of whom $40 \%$ had up-regulated Ki-67 levels, and 13.3\% had down-regulated Ki-67 levels. Kaplan-Meier survival analysis showed that the group with Ki-67 variability had a shorter overall survival $(p=0.0297)$. The Cox regression analysis indicated that Ki-67 variability ( $p=0.038)$ was the only independent prognostic factor for overall survival.

Conclusions: Our data suggest that patients with GEP-NENs and Ki-67 variability had a poorer prognosis. The re-assessment of $\mathrm{Ki}-67$ at sites of metastasis or during the disease course might play a role in predicting the prognosis of patients with GEP-NENs. This finding could have implications for how GEP-NENs are monitored and treated.
\end{abstract}

Keywords: Gastroenteropancreatic neuroendocrine neoplasms, Ki-67, Metastases, Variability, Prognostic factors

\section{Background}

The Ki-67 protein, a cell proliferation-associated nuclear marker, has become a useful tool in assessing the malignant potential of neuroendocrine neoplasms (NENs) [1-3]. With respect to gastroenteropancreatic neuroendocrine neoplasms (GEP-NENs), the Ki-67 labeling index had already become an integral part of the World Health

\footnotetext{
* Correspondence: selinalin35@hotmail.com

${ }^{1}$ Department of Gastroenterology, Union Hospital, Tongji Medical College, Huazhong University of Science and Technology, Wuhan 430022, China Full list of author information is available at the end of the article
}

Organization (WHO) classification, from as early as the 2004 edition [4]. Subsequently, in the WHO-2010 classification schemes, GEP-NENs were further subdivided into three grades by the Ki-67 index as follows: grade 1 (G1) tumors with a Ki-67 index $\leq 2 \%$; (grade 2) G2 tumors with an index of 3-20\%; and (grade 3) G3 tumors, > 20\% [5]. For NENs, a higher Ki-67 labeling index is associated with a poorer prognosis $[2,6,7]$. Accordingly, the grading system based on Ki-67 facilitates identification of the aggressive subset of NENs and provides a standardized

(c) The Author(s). 2018 Open Access This article is distributed under the terms of the Creative Commons Attribution 4.0 International License (http://creativecommons.org/licenses/by/4.0/), which permits unrestricted use, distribution, and reproduction in any medium, provided you give appropriate credit to the original author(s) and the source, provide a link to the Creative Commons license, and indicate if changes were made. The Creative Commons Public Domain Dedication waiver (http://creativecommons.org/publicdomain/zero/1.0/) applies to the data made available in this article, unless otherwise stated. 
diagnostic pathway that is appropriate for effective decisionmaking in the management of NENs.

The GEP-NENs, one kind of heterogeneous tumors, frequently present with metastatic deposits at initial diagnosis; distant metastases have been suggested to be one of the strongest predictors of survival in GEP-NENs $[1,8-10]$. In recent years, several publications have noted that the Ki-67 index varies from the site of the primary tumor to those of metastases, and even throughout the disease course $[1,2,11]$. In addition, researchers have also advocated that a sufficient evaluation of the Ki-67 index in metastatic tumors could have prognostic value and might be necessary to optimize clinical decision-making.

Despite the fact that discordant expression of Ki-67 exists at the primary and metastatic tumor sites, little is known about the manner in which Ki-67 variability changes throughout the disease, and thus affects prognosis. This is partly due to low prevalence and a lack of large sample data on GEP-NENs. We reviewed 514 patients with GEP-NENs and assessed the Ki-67 levels in the subgroup whose specimens bore multiple pathologies. Our aims were to explore the relationship between Ki-67 variability and prognosis in patients with GEP-NENs and provide the basis for more accurate decision-making in GEP-NENs.

\section{Methods}

We conducted a single-center retrospective study. The present study retrospectively reviewed patients diagnosed with GEP-NENs at Wuhan Union Hospital from July 2009 to February 2018. A total of 514 patients were included in the study, among which, 30 had multiple specimens taken from the primary tumor and a metastatic focus, or during the course of the disease. The diagnosis of NENs was performed through conventional histological and immunohistochemical analysis of specimens from the primary tumor and/or metastatic lesions. All specimens obtained by surgical resection, fine needle aspiration, and/or core biopsy were made available for all enrolled patients. The medical records were retrospectively reviewed to collect the following data: age, sex, primary and metastatic tumor sites, and Ki-67 labeling index.

The study was approved by the Ethics Committee of Tongji Medical College, Huazhong University of Science and Technology (IORG No: IORG0003571), and performed in accordance with the Declaration of Helsinki. As it was a retrospective study, all data were collected from a medical records system. Therefore, the study was exempt from the requirement to obtain individual informed consent, based on the Ethical Guidelines of the Ethics Committee of Tongji Medical College, Huazhong University of Science and Technology.

\section{Immunohistochemistry}

The specimens were fixed in $4 \%$ paraformaldehyde and embedded in paraffin wax. To evaluate the Ki-67 proliferation index of tumors, the paraffin-embedded tissue blocks were cut into $4 \mu \mathrm{m}$ thick sections, and tissue sections were then assessed by immunohistochemistry with a Ki-67 antibody (MIB-1, DAKO), using the Ventana Discovery staining system (Ventana Medical Systems). The Ki-67 index was determined by calculating the percentage of tumor cells with positive staining, among up to 2000 tumor cells in the densest field of each slide. All results were verified by two pathologists (QZ and the pathologist responsible for the original pathology report).

\section{Statistical analysis}

Clinical and pathologic characteristics of patients were expressed as median and range, or percentage. Overall survival was defined as that time from the date of diagnosis to the date of death, or last follow-up. Survival curves were drawn according to the Kaplan-Meier analysis, and differences between groups were assessed using the log-rank test. Cox proportional hazards regression analysis was used to assess prognostic factors for survival. Statistical calculations and data manipulation were performed using the SPSS software v21.0 (IBM, USA), and $p<0.05$ was considered statistically significant.

\section{Results}

\section{Patient characteristics}

Among the 514 patients with a GEP-NENS diagnosis, $302(58.75 \%)$ were men and 212 were $(41.25 \%)$ women. The median age at the time of diagnosis was 55 years (range: $12-85$ years). Of the 514 GEP-NENs patients, 196 (38.13\%) cases were of low grade G1; 102 (19.84\%) of intermediate grade G2; and 216 (42.02\%) of high grade G3. Metastases were observed in 35.41\% (182/514) of all cases, and in 9.18\% (18/196) of G1 tumors, 39.22\% (40/102) of G2 tumors, and 57.41\% (124/216) of G3 tumors. The clinical characteristics of these patients and tumors are shown in Table 1. The subset of 30 patients with specimens showing multiple pathologies were analyzed, to evaluate the heterogeneity of the Ki-67 index throughout the disease course (Table 2).

\section{Variability of Ki-67 throughout the disease course of NENs} Among the 30 patients, 10 (33.3\%) were G1; 7 (23.3\%) G2; and 13 (43.3\%) G3. Assessment of the Ki-67 index in those 30 cases with specimens showing multiple pathologies revealed discrepancies in $53.3 \%$ cases, among which, $40 \%$ and 13.3\% patients had up-regulated and down-regulated Ki-67 levels, respectively. The up-regulation of the Ki-67 index from primary to metastatic specimens, or during the disease course was as follows: G1 to G2, 25.0\% (4/16); G2 to 
Table 1 Clinical characteristics in 514 patients with GEP-NENs

\begin{tabular}{ll}
\hline Variables & Total $n=514(\%)$ \\
\hline Sex & $302(58.75)$ \\
Male & $212(41.25)$ \\
Grade (Ki-67) & \\
G1 & $196(38.13)$ \\
G2 & $102(19.84)$ \\
G3 & $216(42.02)$ \\
Primary tumor site & \\
Pancreas & $149(28.99)$ \\
Large colon & $148(28.79)$ \\
Stomach & $100(19.46)$ \\
Esophagus & $22(4.28)$ \\
Duodenum & $16(3.11)$ \\
Other sites & $79(15.37)$ \\
Metastasis & $182(35.41)$ \\
Age at diagnosis (years) & $55(12-85)$ \\
\hline
\end{tabular}

G3, $6.25 \%$ (1/16); G2 to G2, $6.25 \%$ (1/16); G3 to G3, $37.5 \%$ (6/16).

Some Ki-67 variability was observed in $41.18 \%$ (7/17) of the patients with primary tumors categorized as G1/G2; $35.29 \%(6 / 17)$ showed up-regulation of Ki-67 and 5.88\% (1/17) showed down-regulation of Ki-67 (Fig. 1a). For primary tumors categorized as G3 (as confirmed by both $\mathrm{Ki}-67$ and mitotic count), the Ki-67 variability was $57.1 \%$ (9/13), including $46.15 \%$ (6/13) showing up-regulation, and $23.08 \%$ (3/13) showing down-regulation (Fig. 1a).

The rectum was the most variable of the primary sites, whereas grade 3 (G3) tumors had the most variable intervals. Among the 11 patients with primary tumors in the rectum, Ki-67 variability was present in $72.72 \%(8 / 11)$ of all cases; $54.54 \%$ (6/11) showed up-regulation, and $18.18 \%(2 / 11)$ showed down-regulation (Fig. 1b). About $28.6 \%$ of the patients with primary tumors in the pancreas had up-regulated Ki-67 levels in metastatic loci and were upstaged to a higher WHO class (from G2 to G3) (Table 2, Fig. 1b). For ten patients with primary tumors in the stomach, $60 \%$ (6/10) showed Ki-67 variability, with $40 \%$ (4/10) showing up-regulation, and 20\% (2/10) showing down-regulation of Ki-67 levels (Fig. 1b).

\section{Survival analysis}

Kaplan-Meier survival analysis showed a significant discrepancy in mortality between Ki-67 variable and non-variable groups; the group with Ki-67 variability had a poorer prognosis than the group without Ki-67 variability ( $p=0.0297)$ (Fig. 2). Cox regression analysis included age, sex, primary and metastatic tumor sites, Ki-67 level, and Ki-67 variability, and showed that Ki-67 variability $(p=0.038)$ was the only prognostic factor for survival in patients with metastatic GEP-NENs (Table 3).

\section{Discussion}

In this study, we explored the relationship between the Ki-67 variability and the prognosis of patients with GEP-NENs. Our data support the fact that the Ki-67 index in GEP-NENs can change throughout the disease course, often with progression to increased malignancy and greater aggressiveness after metastasis. Moreover, this study further demonstrates that patients with Ki-67 variability have a poorer prognosis. Thus, re-assessment of Ki-67 at the sites of metastases, or during the disease course may prove to be a significant step in determining the prognosis of patients with GEP-NENs.

The GEP-NENs represent a heterogeneous family with variable biological and clinical characteristics [7, 12, 13]. Over the last few decades, neuroendocrine tumors (NETs) have been commonly considered rare tumors. However, the real incidence and prevalence of NETs, that has increased 6.4-fold from 1973 (1.09 per $100,000)$ to 2012 (6.98 per 100,000), according to Surveillance, Epidemiology, and End Results (SEER) data, may be underestimated [14].

Although the role of the Ki-67 index in GEP-NENs has been widely recognized since 2004, some recent studies have found that the Ki-67 index might change throughout the disease course or between primary and metastatic sites $[1,2,11,15]$. One UK study showed that Ki-67 variability existed in $41.2 \%$ GEP-NEN cases from the primary tumor to metastatic sites [1]. Singh et al. (2014) proposed that Ki-67 might vary during the disease course, from primary stage to metastasis, and these changes throughout the course of the disease might have a significant impact on the monitoring and management of NETs [2]. Along with those observations, we also found that variability of the $\mathrm{Ki}-67$ index between primary and metastatic specimens, or during the disease course was identified in about $53.3 \%$ of the patients in the present study.

We further analyzed the cases with Ki-67 index variability. Among 30 patients, the Ki-67 levels were found to be upand down-regulated in $40 \%$ and $13.3 \%$ of the cases, respectively from the primary site to the metastatic site or during the disease course. The up-regulation of Ki-67 levels was as follows: G1 to G2, 25.0\%; G2 to G3, 6.25\%; G3 to G3, 37.5\%. Shifting to a higher grade was mainly observed between G1 and G2, and as both G1 and G2 tumors received the same treatment, the clinical management based on the current criteria was not affected $[16,17]$. In about $6.25 \%$ of the patients showing Ki-67 index variability, the tumor grades were upstaged from G2 to G3. However, without re-assessments of the metastasis or during the disease 
Table 2 Ki-67 index variability and WHO class change in patients with multiple pathology specimens

\begin{tabular}{|c|c|c|c|c|c|c|}
\hline \multirow[t]{2}{*}{ Patient \# } & \multirow{2}{*}{$\begin{array}{l}\text { Primary tumor } \\
\text { site Site1\# }\end{array}$} & \multirow{2}{*}{$\begin{array}{l}\text { Metastatic/Re-biopsy } \\
\text { tumor site Site2\# }\end{array}$} & \multicolumn{2}{|c|}{ Ki-67 index (\%) } & \multirow{2}{*}{$\begin{array}{l}\text { Survival } \\
\text { time }\end{array}$} & \multirow{2}{*}{$\begin{array}{l}\text { WHO class } \\
\text { change }\end{array}$} \\
\hline & & & Site1\# & Site2\# & & \\
\hline 1 & pancreas & peritoneum & 70 & 70 & 12 & - \\
\hline 2 & pancreas & lymph node & 10 & 30 & 15 & $\mathrm{G} 2 \rightarrow \mathrm{G} 3$ \\
\hline 3 & pancreas & peritoneum & 5 & 20 & 6 & $\mathrm{G} 2 \rightarrow \mathrm{G} 2$ \\
\hline 4 & pancreas & liver & 5 & 5 & 11 & - \\
\hline 5 & pancreas & liver & 2 & 2 & 12 & - \\
\hline 6 & pancreas & pancreas & 1 & 1 & 24 & - \\
\hline 7 & pancreas & liver & 1 & 1 & 38 & - \\
\hline 8 & rectum & lymph node & 70 & 70 & 12 & - \\
\hline 9 & rectum & liver & 50 & 80 & 16 & $\mathrm{G} 3 \rightarrow \mathrm{G} 3$ \\
\hline 10 & rectum & liver & 2 & 5 & 13 & $\mathrm{G} 1 \rightarrow \mathrm{G} 2$ \\
\hline 11 & rectum & liver & 2 & 1 & 5 & $\mathrm{G} 1 \rightarrow \mathrm{G} 1$ \\
\hline 12 & rectum & liver & 2 & 5 & 3 & $\mathrm{G} 1 \rightarrow \mathrm{G} 2$ \\
\hline 13 & rectum & lymph node & 70 & 90 & 8 & $\mathrm{G} 3 \rightarrow \mathrm{G} 3$ \\
\hline 14 & rectum & lymph node & 2 & 5 & 7 & $\mathrm{G} 1 \rightarrow \mathrm{G} 2$ \\
\hline 15 & rectum & liver & 2 & 2 & 14 & - \\
\hline 16 & rectum & liver & 10 & 10 & - & - \\
\hline 17 & rectum & rectum & 80 & 60 & 12 & $\mathrm{G} 3 \rightarrow \mathrm{G} 3$ \\
\hline 18 & rectum & lymph node & 60 & 80 & - & $\mathrm{G} 3 \rightarrow \mathrm{G} 3$ \\
\hline 19 & stomach & lymph node & 2 & 5 & 12 & $\mathrm{G} 1 \rightarrow \mathrm{G} 2$ \\
\hline 20 & stomach & lymph node & 60 & 80 & 14 & $\mathrm{G} 3 \rightarrow \mathrm{G} 3$ \\
\hline 21 & stomach & liver & 1 & 1 & 24 & - \\
\hline 22 & stomach & lymph node & 60 & 40 & 22 & $\mathrm{G} 3 \rightarrow \mathrm{G} 3$ \\
\hline 23 & stomach & lymph node & 5 & 5 & 27 & - \\
\hline 24 & stomach & stomach & 50 & 80 & - & $\mathrm{G} 3 \rightarrow \mathrm{G} 3$ \\
\hline 25 & stomach & stomach & 70 & 30 & 33 & $\mathrm{G} 3 \rightarrow \mathrm{G} 3$ \\
\hline 26 & stomach & stomach & 30 & 40 & 21 & $\mathrm{G} 3 \rightarrow \mathrm{G} 3$ \\
\hline 27 & stomach & pancreas & 70 & 70 & 8 & - \\
\hline 28 & stomach & stomach & 5 & 5 & 60 & - \\
\hline 29 & ileocecal junction & lymph node & 70 & 70 & 16 & - \\
\hline 30 & duodenum & liver & 5 & 5 & 12 & - \\
\hline
\end{tabular}
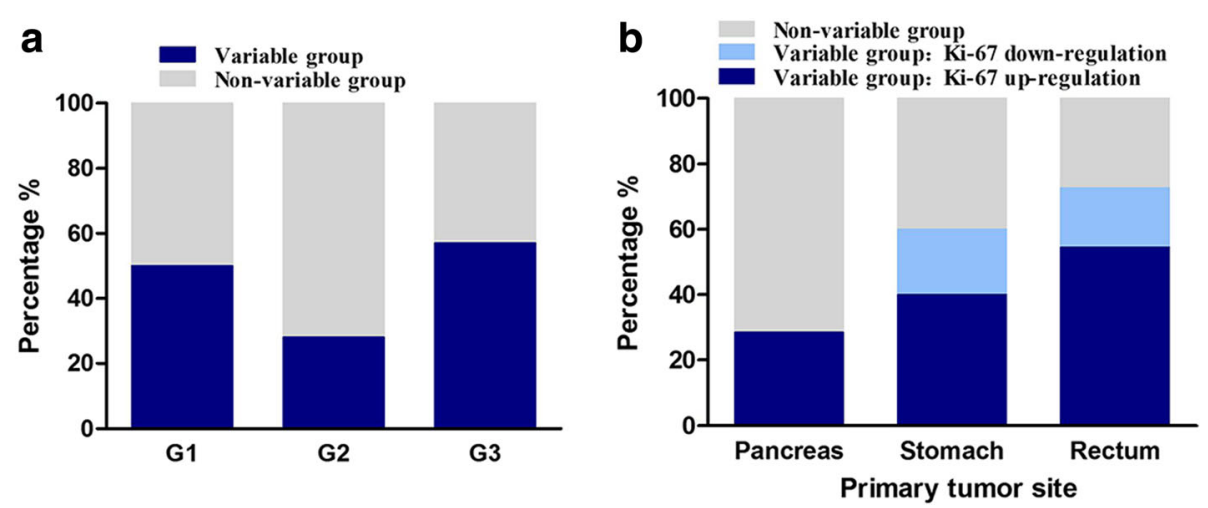

Fig. 1 Distribution of variable cases according to GEP-NENs grade (a) and primary tumor site (b) 


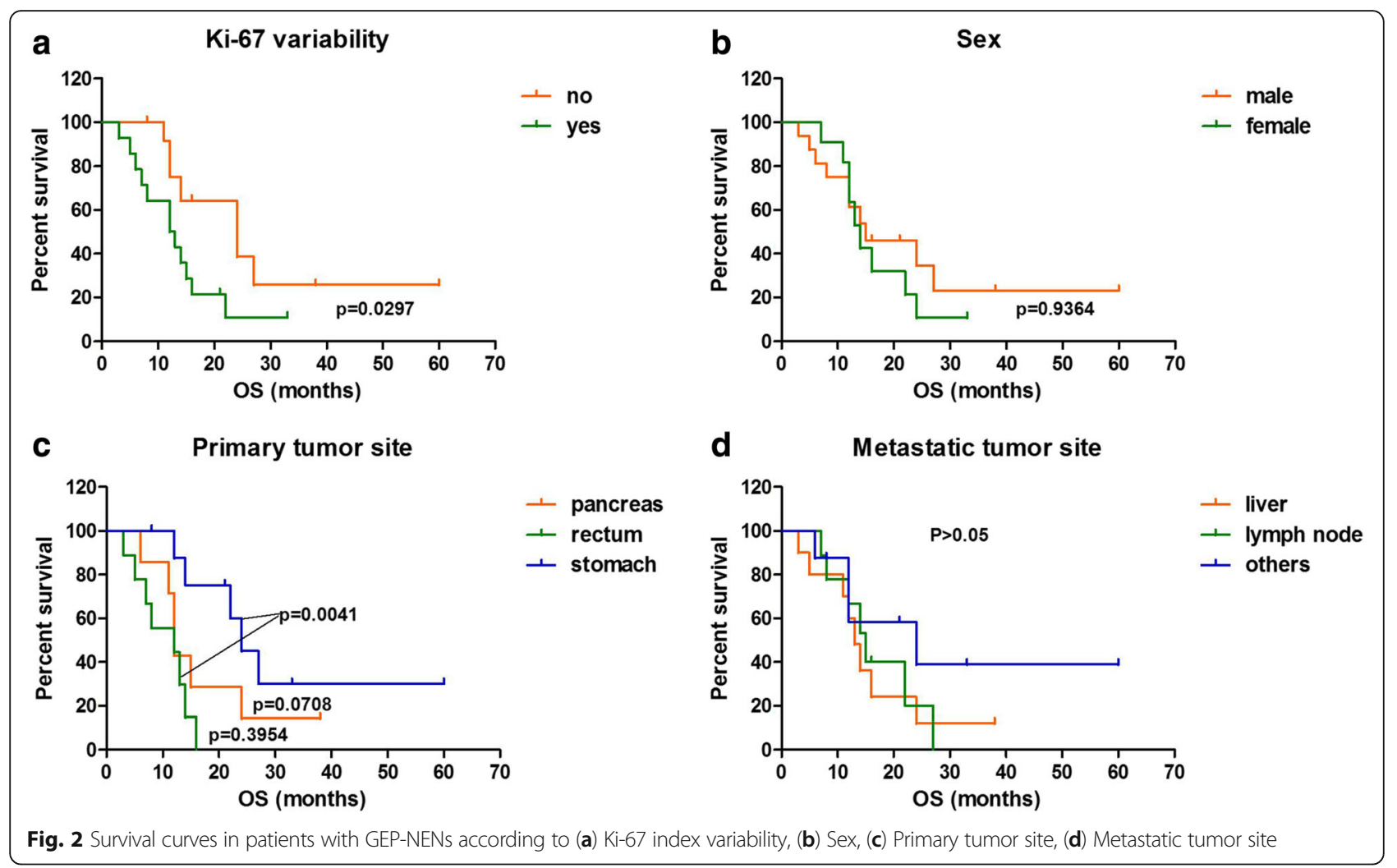

course, those G1/G2 NENs might not have been recognized as potential candidates for chemotherapy. Therefore, our results suggest that identification of the upstaged subset of G1/G2 was significantly useful to clinical doctors in determining appropriate treatment options and evaluating prognosis.

Although previous papers $[1,2,11]$ have advocated re-assessment of the Ki-67 index throughout the disease course, or the progression from primary to metastatic sites, reports on whether variability in Ki-67 levels affects the prognosis of GEP-NENs are lacking. Kaplan-Meier survival analysis of the patients of the present study showed that the group with Ki-67 variability had a significantly shorter overall survival. Furthermore, results of the Cox regression analysis further confirmed that Ki-67 variability was the only independent prognostic factor for survival in those patients. These results emphasize the need for biopsies from metastatic lesions, or over the course of the disease. Moreover, the assessment of Ki-67 levels at all sites could significantly improve patient management.

To date, the reasons for $\mathrm{Ki}-67$ index variability in GEP-NENs remain unclear. Miller et al. [1] hypothesized that variation of Ki-67 expression within a tumor is due to genetic intramural heterogeneity of NENs, as had been shown in other solid cancers [18-20]. However, Singh et al. [2] supposed that Ki-67 index changes during

Table 3 Cox regression analysis on prognostic baseline factors for survival in patients with multiple pathology specimens

\begin{tabular}{|c|c|c|c|c|}
\hline \multirow[t]{2}{*}{ Characteristics } & \multicolumn{2}{|l|}{ Univariate analysis } & \multicolumn{2}{|c|}{ Multivariate Cox regression analysis } \\
\hline & HR $(95 \% \mathrm{Cl})$ & $P$ value & $\mathrm{HR}(95 \% \mathrm{Cl})$ & $P$ value \\
\hline Age & $0.994(0.954-1.037)$ & 0.789 & & \\
\hline Sex, female vs. Male & $1.312(0.530-3.248)$ & 0.559 & & \\
\hline Primary tumor site & & 0.068 & & 0.310 \\
\hline Pancreas vs. Stomach & $2.379(0.720-7.852)$ & 0.155 & $2.031(0.564-7.311)$ & 0.278 \\
\hline Rectum vs. Stomach & $5.468(1.570-19.041)$ & 0.008 & $3.320(0.956-11.523)$ & 0.059 \\
\hline Metastasis, Yes vs. No & $6.142(0.810-46.551)$ & 0.079 & $6.963(0.814-59.588)$ & 0.076 \\
\hline Ki-67 index & $0.995(0.979-1.011)$ & 0.565 & & \\
\hline Ki-67 Variability, Yes vs. No & $2.612(0.999-6.834)$ & 0.045 & $3.487(1.069-11.380)$ & 0.038 \\
\hline
\end{tabular}


the disease course could be due to treatment effects and therapy resistance. Although the present study showed that the Ki-67 index varies from primary to metastatic sites, or during the disease course, we were not able to draw solid conclusions about the reasons behind the Ki-67 variability observed because of the relatively small sample size. The underlying mechanisms need to be explored in further studies with larger sample sizes.

\section{Conclusions}

In summary, our study confirms that discordant expressions of Ki-67 in primary tumors and metastases are common in GEP-NENs. Furthermore, we also presented strong evidence that patients with Ki-67 variability have a poorer prognosis in GEP-NENs, and there is need for increased vigilance with this subgroup. Therefore, we recommend as a matter of great importance, a re-biopsy and re-estimation of the Ki-67 index at metastatic sites, or during the disease course. Although both intra-tumor heterogeneity and therapy resistance are speculated to be the underlying mechanisms of Ki-67 variability, the manner in which they affect patient prognosis requires further study.

\section{Abbreviations}

GEP-NENs: Gastroenteropancreatic Neuroendocrine Neoplasms; NETs: Neuroendocrine tumors; OS: Overall survival; SEER: Surveillance, Epidemiology, and End Results program; WHO: World Health Organization

\section{Acknowledgements}

The authors wish to acknowledge all participants in this study and everybody involved in the set-up and implementation of the study.

\section{Funding}

This study was supported by the National Natural Science Foundation of China (Nos. 81572428 and 81770539).

\section{Availability of data and materials}

The datasets used and/or analysed during the current study are available from the corresponding author on reasonable request.

\section{Authors' contributions}

$\mathrm{RL}$ and HYS designed/performed most of the investigation, data analysis and wrote the manuscript; QZ provided pathological assistance; $\mathrm{CQH}$ and ZD contributed to interpretation of the data and analyses. All of the authors have read and approved the manuscript.

\section{Ethics approval and consent to participate}

This study was approved by the ethics committee of Tongji Medical College, Huazhong University of Science and Technology (IORG No: IORG0003571), and performed in accordance with the Declaration of Helsinki. As it was a retrospective study, all data were collected from a medical records system. Therefore, the study was exempt from the requirement to obtain individual informed consent, based on the Ethical Guidelines of the Ethics Committee of Tongji Medical College, Huazhong University of Science and Technology.

\section{Consent for publication}

Not applicable.

\section{Competing interests}

The authors declare that they have no competing interests.

\section{Publisher's Note}

Springer Nature remains neutral with regard to jurisdictional claims in published maps and institutional affiliations.

\section{Author details}

${ }^{1}$ Department of Gastroenterology, Union Hospital, Tongji Medical College, Huazhong University of Science and Technology, Wuhan 430022, China. ${ }^{2}$ Department of Pathology, Union Hospital, Tongji Medical College, Huazhong University of Science and Technology, Wuhan 430022, China.

Received: 4 April 2018 Accepted: 27 June 2018

Published online: 28 July 2018

\section{References}

1. Miller HC, Drymousis P, Flora R, Goldin R, Spalding D, Frilling A. Role of Ki-67 proliferation index in the assessment of patients with neuroendocrine neoplasias regarding the stage of disease. World J Surg. 2014;38(6):1353-61.

2. Singh S, Hallet J, Rowsell C, Law CH. Variability of Ki67 labeling index in multiple neuroendocrine tumors specimens over the course of the disease. European journal of surgical oncology : the journal of the European Society of Surgical Oncology and the British Association of Surgical Oncology. 2014; 40(11):1517-22

3. Foltyn W, Zajecki W, Marek B, Kajdaniuk D, Sieminska L, Zemczak A, KosKudla B. The value of the Ki-67 proliferation marker as a prognostic factor in gastroenteropancreatic neuroendocrine tumours. Endokrynologia Polska. 2012;63(5):362-6.

4. Kleihues PSL. World Health Organization classification of Tumours. Pathology and genetics of endocrine organs. In: Lyon: IARC press; 2004

5. Bosman FT CF, Hruban RH et al: WHO Classification of Tumours of the Digestive System, 4th edition. Geneva, Switzerland: WHO Press 2010, ISBN978-92-832-2432-7.

6. Khan MS, Luong TV, Watkins J, Toumpanakis C, Caplin ME, Meyer T. A comparison of $\mathrm{Ki}-67$ and mitotic count as prognostic markers for metastatic pancreatic and midgut neuroendocrine neoplasms. Br J Cancer. 2013;108(9): 1838-45.

7. Adsay V. Ki67 labeling index in neuroendocrine tumors of the gastrointestinal and pancreatobiliary tract: to count or not to count is not the question, but rather how to count. Am J Surg Pathol. 2012;36(12):1743-6.

8. Yao JC, Hassan M, Phan A, Dagohoy C, Leary C, Mares JE, Abdalla EK, Fleming JB, Vauthey JN, Rashid A, et al. One hundred years after "carcinoid": epidemiology of and prognostic factors for neuroendocrine tumors in 35,825 cases in the United States. Journal of clinical oncology : official journal of the American Society of Clinical Oncology. 2008;26(18):3063-72.

9. Lewkowicz E, Trofimiuk-Muldner M, Wysocka K, Pach D, Kieltyka A, Stefanska A, Sowa-Staszczak A, Tomaszewska R, Hubalewska-Dydejczyk A.

Gastroenteropancreatic neuroendocrine neoplasms: a 10-year experience of a single center. Polskie Archiwum Medycyny Wewnetrznej. 2015;125(5):337-46.

10. Scarpa A, Mantovani W, Capelli P, Beghelli S, Boninsegna L, Bettini R, Panzuto F, Pederzoli P. Delle Fave G, Falconi M. pancreatic endocrine tumors: improved TNM staging and histopathological grading permit a clinically efficient prognostic stratification of patients. Modern pathology: an official journal of the United States and Canadian Academy of Pathology, Inc. 2010;23(6):824-33.

11. Grillo F, Albertelli M, Brisigotti MP, Borra T, Boschetti M, Fiocca R, Ferone D, Mastracci L. Grade increases in Gastroenteropancreatic neuroendocrine tumor metastases compared to the primary tumor. Neuroendocrinology. 2016;103(5):452-9.

12. Yang $Z$, Tang LH, Klimstra DS. Gastroenteropancreatic neuroendocrine neoplasms: historical context and current issues. Semin Diagn Pathol. 2013; 30(3):186-96.

13. Cives M, Soares HP, Strosberg J. Will clinical heterogeneity of neuroendocrine tumors impact their management in the future? Lessons from recent trials. Curr Opin Oncol. 2016;28(4):359-66.

14. Dasari A, Shen C, Halperin D, Zhao B, Zhou S, Xu Y, Shih T, Yao JC. Trends in the incidence, prevalence, and survival outcomes in patients with neuroendocrine tumors in the United States. JAMA oncology. 2017;3(10):1335-42

15. Dhall D, Mertens R, Bresee C, Parakh R, Wang HL, Li M, Dhall G, Colquhoun $\mathrm{SD}$, Ines D, Chung F, et al. Ki-67 proliferative index predicts progression-free survival of patients with well-differentiated ileal neuroendocrine tumors. Hum Pathol. 2012;43(4):489-95

16. Modlin IM, Oberg K, Chung DC, Jensen RT, de Herder WW, Thakker RV, Caplin M, Delle Fave G, Kaltsas GA, Krenning EP, et al. Gastroenteropancreatic neuroendocrine tumours. The Lancet Oncology. 2008;9(1):61-72. 
17. Pape UF, Perren A, Niederle B, Gross D, Gress T, Costa F, Arnold R, Denecke T, Plockinger U, Salazar R, et al. ENETS consensus guidelines for the management of patients with neuroendocrine neoplasms from the jejuno-ileum and the appendix including goblet cell carcinomas. Neuroendocrinology. 2012;95(2): $135-56$.

18. Kumler I, Balslev E, Knop AS, Brunner N, Klausen TW, Jespersen SS, Nielsen $\mathrm{SL}$, Nielsen DL. Expression patterns of biomarkers in primary tumors and corresponding metastases in breast Cancer. In: Applied immunohistochemistry \& molecular morphology : AIMM; 2016.

19. Yamazaki Y, Nakamura Y, Shibahara Y, Konosu-Fukaya S, Sato N, KubotaNakayama F, Oki Y, Baba S, Midorikawa S, Morimoto R, et al. Comparison of the methods for measuring the Ki-67 labeling index in adrenocortical carcinoma: manual versus digital image analysis. Hum Pathol. 2016;53:41-50.

20. Valous NA, Lahrmann B, Halama N, Bergmann F, Jager D, Grabe N. Spatial intratumoral heterogeneity of proliferation in immunohistochemical images of solid tumors. Med Phys. 2016;43(6):2936-47.

Ready to submit your research? Choose BMC and benefit from:

- fast, convenient online submission

- thorough peer review by experienced researchers in your field

- rapid publication on acceptance

- support for research data, including large and complex data types

- gold Open Access which fosters wider collaboration and increased citations

- maximum visibility for your research: over $100 \mathrm{M}$ website views per year

At BMC, research is always in progress.

Learn more biomedcentral.com/submissions 